\title{
Tenascin-C (TNC) Promotes Breast Cancer Cell Invasion and Proliferation: Functional Effects of TNC Knockdown in Highly Invasive Breast Cancer Cell Lines
}

\author{
Ali S Alharth ${ }^{1, *}$, Musaad A Alsulaiman ${ }^{1}$, Waleed A Alyami ${ }^{2}$ \\ ${ }^{1}$ Department of Public Health, Ministry of Health, Riyadh, Saudi Arabia \\ ${ }^{2}$ General Health Affairs, Ministry of Health, Riyadh, Saudi Arabia \\ *Corresponding author: alharth_a@yahoo.com
}

Received April 02, 2015; Revised April 18, 2015; Accepted April 21, 2015

\begin{abstract}
The tumour microenvironment plays a crucial role in the development of breast cancer. Tenascin-C (TNC), a matricellular protein and its high molecular weight (MW) isoforms have been shown to be over-expressed in the stroma of breast cancers and are associated with poor prognosis. The aim of this study was to investigate the effects of TNC knockdown in TNC expressing invasive breast cancer cell lines on cancer cell behaviour. Small interfering RNA (siRNA) targeting different exons in TNC (24, 14 and 14-AD1) were designed, synthesised and transfected into the highly invasive MDA-MB-231 breast cancer cell line. The phenotypic alterations caused by TNC knockdown were analysed by Two Dimension (2D) invasion assays and proliferation assays using the mitotic marker Phispho-Histone H3 (pHH3). The siRNA targeted cells showed significant down-regulation of both total TNC ( $\mathrm{p}<0.001)$ and high MW isoforms ( $\mathrm{p}<0.001)$ in MDA-MB-231 cells. Moreover, knockdown of total TNC and high MW TNC isoforms significantly decreased both cell invasion (total TNC $\mathrm{p}<0.001$ and TNC-14 $\mathrm{p}<0.001$ ) and proliferation (total TNC $\mathrm{p}<0.001$ and TNC-AD1 $\mathrm{p}<0.05$ ). In conclusion, TNC knockdown significantly decreases proliferation and invasion in breast cancer cell lines, confirming its importance in breast cancer progression.
\end{abstract}

Keywords: TNC, siRNA, knockdown

Cite This Article: Ali S Alharth, Musaad A Alsulaiman, and Waleed A Alyami, "Tenascin-C (TNC) Promotes Breast Cancer Cell Invasion and Proliferation: Functional Effects of TNC Knockdown in Highly Invasive Breast Cancer Cell Lines.” American Journal of Medical and Biological Research, vol. 3, no. 2 (2015): 55-61. doi: 10.12691/ajmbr-3-2-2.

\section{Introduction}

Breast cancer is one of the most common cancers in the world with high mortality and morbidity rates, complicated by unique molecular signatures and diverse genetic alterations; each with distinct clinical outcomes [1]. The microenvironment of the tumour includes cells and extracellular matrix (ECM), with interactions between tumour cells and the surrounding microenvironment being important for proliferation, survival, differentiation and migration. Alterations in the tumour microenvironment have been shown to play a crucial role in breast cancer progression ([2,3]). Although many ECM proteins are altered in breast cancer, one consistent finding was the upregulation of the hexameric protein tenascin-C (TNC) in the tumour stroma $([4,5])$.

TNC is an oligomeric ECM glycoprotein with a molecular mass of $180-300 \mathrm{kDa}$ that contains six monomers linked by disulfide bonds at their $\mathrm{N}$-termini [6]. Alternative splicing at the pre-mRNA level of 9 FNIII repeats (exons 10-16, AD1 and AD2), which are included or excluded in a unique manner, allows TNC to show tremendous diversity in isoform expression with as many as 27 different mRNA variants having been identified in the developing mouse brain [7].

TNC expression has been shown to be increased in cancers including carcinomas of the colon, breast, lung, prostate and brain [8]. Expression of TNC is also associated with poor prognosis and has been shown to predict local and distant recurrence in breast cancer [9], as well as being been correlated with high HER2, low oestrogen receptor (ER) expression and lymph node metastasis [10].

TNC has been shown to promote cell migration, inhibit focal adhesion formation, induce cell proliferation, and act as a cell survival factor as well as promoting angiogenesis and remodelling of ECM components such as Matrix metalloproteinases (MMPs) [11]. The variability in expression of TNC exons has been shown to play a crucial role in the progression of breast cancer. Adams [12] examined in detail the pattern of TNC isoform expression in benign, pre-invasive and invasive breast lesions using reverse transcription - polymerase chain reaction (RTPCR) and Southern blotting, with a distinct diversity in the expression of TNC isoforms in breast cancer being observed. Although fully truncated TNC was identified in all breast cancer tissues, isoforms containing exons $14+$ 16 (TNC14/16) were significantly associated with an 
invasive phenotype. Highly invasive breast cancer cell lines such as MDA-MB 231 and MDA-MB-436 were shown to express TNC, whereas less invasive, ER positive cell lines such as MCF-7 and T47D showed no detectable levels of TNC. Moreover, cell invasion and proliferation were promoted by over-expression of TNC16 and TNC 14/16 in MCF-7, T47D, MDA-MD-231, MDA-MB-468 and GI101 breast cancer cell lines via MMP dependent and independent mechanisms [13].

Expression of isoforms containing additional domain 1 (AD1) were found to be significantly associated with high grade, ER negative tumours as well as younger patient age (<40 years) [14].

Knockdown of TNC using short hairpin RNA showed a significant reduction invasion and metastasis of breast cancer cells to the lung [15]. In glioblastoma, TNC was down regulated by using of small interfering RNA (siRNA) and resulted in a significant decrease in metastasis [16], and decreased invasiveness and reactive change of peritumoral brain tissue [17]. In melanoma, TNC knockdown decreased the side population (SP) fraction in melanoma spheres and lowered their resistance to doxorubicin treatment [18]. Knowledge of TNC knockdown using siRNA technology in breast cancer is limited. However, previous findings suggest that knockdown of endogenous TNC using siRNA plays a crucial role in tumour invasion and metastases. In addition, as specific TNC isoforms have been shown to influence breast cancer cell behaviour.
The aims of the present study were to knockdown total TNC and high MW TNC isoforms (TNC-14 and TNC-14AD1) in highly invasive breast cancer cell lines. To determine the effect of TNC knockdown on tumour cell behaviour. The results of the study show that TNC silencing significantly reduced cancer cell proliferation and invasion confirming its crucial role in cancer progression.

\section{Materials and Methods}

\subsection{Cell Lines}

Breast cell lines (MDA-MB-231, MDA-MB-468, MDA-MB-436 and HBL-100) were obtained from American Type Culture Collection (Rockville, MD, USA). MDA-MB-436cell line were grown in RPMI with $10 \%$ FBS. Other cell lines were grown in DMEM containing 2 mM L-glutamine and 10\% FBS (Invitrogen Life Science, Carlsbad, CA, USA).

\subsection{Transfection of MDA-MB-231 Breast Carcinoma Cell Lines with siRNA}

Breast cancer cell lines MDA-MB-231 were transfected with synthetic siRNA targeting total TNC and TNC isoforms (Table 1), and scrambled siRNA as a negative control. Lipofectamine 2000 transfection reagent (Invitrogen, UK) was used according to the manufacturing protocol. The assessment of the transfection efficiency was performed using fluorescently labelled siRNA.

Table 1. Summary of siRNA used in the study

\begin{tabular}{|l|l|c|c|}
\hline Target Name & Sense \& Antisense Strands Sequences & Length & Region \\
\hline Total TNC & $\begin{array}{l}\text { 5'CGCGAGAACUUCUACCAAAtt3' } \\
\text { 5'UUUGGUAGAAGUUCUCGCGtc3' }\end{array}$ & 21 & 21 \\
\hline TNC-14 & $\begin{array}{l}\text { 5'CCGAUGGGAUCUUCGAGACUU3' } \\
\text { 5'AAGUCUCGAAGAUCCCAUCGG3' }\end{array}$ & 24 \\
\hline TNC-14-AD1 & $\begin{array}{l}\text { 5'CACGACAGAACCAAAGCCAUU3' } \\
\text { 5'UGGCUUUGGUUCUGUCGUGUU3' }\end{array}$ & 21 & Exon 14 \\
\hline -ve Control & Unknown & 21 & Unknown \\
\hline
\end{tabular}

\subsection{Total RNA Extraction and cDNA Generation}

RNA was extracted from breast cancer cell pellets resuspended in Tri Reagent. mRNA was isolated using
oligo-dT Dynabeads ${ }^{\circledR}$ (Dynal, Bromborough, UK) and reverse transcription was performed at $42^{\circ} \mathrm{C}$ for 1 hour using Expand-RT (Boehringer Mannheim, Welwyn Garden City, UK) as described previously [12].

Table 2. Summary of primers and probes sequences

\begin{tabular}{|c|c|c|c|c|c|}
\hline Primer & Sequence $5^{\prime} \rightarrow 3^{\prime}$ & $\begin{array}{c}\text { Length } \\
\text { (nt) }\end{array}$ & $\begin{array}{l}\text { Tm } \\
\left({ }^{\circ} \mathrm{C}\right)\end{array}$ & $\begin{array}{c}\text { Annealing position } \\
\left(5^{\prime} \rightarrow 3^{\prime}\right)\end{array}$ & Exon \\
\hline qAD1 F & TGG TGG AGA ACA CTG GCT ATG AC & 23 & 60.8 & $187 \rightarrow 209$ & AD1 \\
\hline qAD1 R & GGG ATC CCC AGC CAA GGT & 18 & 61.2 & $245 \rightarrow 228$ & $\mathrm{AD} 1$ \\
\hline qAD1 Probe & CAG TGT GGC AGG AAC & 15 & 70.1 & $212 \rightarrow 226$ & AD1 \\
\hline $\mathrm{qAD} 2 \mathrm{~F}$ & GAT CAC CCC CAT GAG ACC AT & 20 & 58.6 & $121 \rightarrow 140$ & $\mathrm{AD} 2$ \\
\hline qAD2 R & TGA TGA CAG AGC TGC GAG ACA & 21 & 59.4 & $181 \rightarrow 161$ & $\mathrm{AD} 2$ \\
\hline qAD2 Probe & TGC TGT CTG TGC CTG G & 16 & 69.9 & $143 \rightarrow 158$ & $\mathrm{AD} 2$ \\
\hline $\mathrm{q} 14 / 16 \mathrm{~F}$ & TCC GGA CCA AAA CCA TCA GT & 20 & 59.3 & $4679 \rightarrow 4698$ & 14 \\
\hline $\mathrm{q} 14 / 16 \mathrm{R}$ & TGA AAC CAG AAG GTT GTC AAC TTC & 24 & 58.7 & $5022 \rightarrow 4999$ & 16 \\
\hline q14/16 Probe & ACG ACA GAA GCC GAA CC & 17 & 70.3 & $4708 \rightarrow 4714$ and $4988 \rightarrow 4997$ & $14 / 16$ \\
\hline $\mathrm{q} 9 / 16 \mathrm{~F}$ & CAA GCC CGC ACA TGT GAA & 18 & 59.6 & $3321 \rightarrow 3338$ & 9 \\
\hline q9/16 R & TGA AAC CAG AAG GTT GTC AAC TTC & 24 & 58.9 & $5022 \rightarrow 4999$ & 16 \\
\hline q9/16 Probe & ATC CAC TGA AGC CGA AC & 17 & 70.4 & $3342 \rightarrow 3349$ and $4988 \rightarrow 4996$ & $9 / 16$ \\
\hline TNC-S F & GAGCAAGCCCGCACATG & 17 & 58 & $3318 \rightarrow 3334$ & 9 \\
\hline TNC-S R & CCGAATTTTCAGTGATGTCTGAGA & 24 & 59 & $5311 \rightarrow 5288$ & 17 \\
\hline TNC-S Probe & CATCCACTGCCATGGG & 16 & 68 & $3341 \rightarrow 3349$ and $5261 \rightarrow 5267$ & $9 / 17$ \\
\hline Total TNC & Applied Biosystems (Hs01115654_m1) & -- & -- & start 5380 & $17 \rightarrow 18$ \\
\hline HPRT1 & Applied Biosystems (Hs99999909_m1) & -- & -- & start 648 & $6 \rightarrow 7$ \\
\hline
\end{tabular}




\subsection{Quantitative Polymerase Chain Reaction}

Total TNC expression was analysed using inventoried Taqman assays, whereas TNC isoforms expression was analysed using primers and probes designed in house by the previous group $([13,14])$ (Table 2$)$. The reaction for the inventoried Taqman assays was performed using $4 \mu \mathrm{l}$ cDNA, $0.5 \mu \mathrm{l}$ ultra-pure $\mathrm{H} 2 \mathrm{O}, 0.5 \mu \mathrm{l}$ probe, $5 \mu \mathrm{l}$ of $2 \times$ Taqman Fast PCR mastermix. The reaction for TNC isoforms was performed using 3.6 $\mu \mathrm{l}$ cDNA, $0.6 \mu \mathrm{l}$ forward primer, $0.6 \mu \mathrm{l}$ forward primer, $0.2 \mu \mathrm{l}$ probe and 5 $\mu \mathrm{l}$ of $2 \times$ Taqman Fast PCR mastermix. The expression of TNC profiles was calculated using number of molecules in a known concentration of sample. A log2 value was then produced from the mean Ct value and normalised against the mean Ct values for the endogenous controls [14]. The relative expression (RE) of TNC knockdown was calculated relatively to the scrambled siRNA (negative control) after normalisation to the $\mathrm{Ct}$ value for hypoxanthine phosphoribosyltransferase 1 (HPRT1) using the formula : $\mathrm{RE}=2^{-(\Delta \Delta \mathrm{Ct})}$.

\subsection{Western Blot}

Western blot was performed to determine the amount of intra and extracellular levels of total TNC and TNC isoforms. 24 hours post transfection; completed media was replaced with serum-free media and cells were incubated for 48 hours. The concentration of the collected cell lysate and conditioned media was measured using the bovine serum albumin protein assay. Protein was run on 6\% SDS-PAGE gels and transferred to Hybond ECL nitrocellulose membrane (Amersham Biosciences, Little Chalfont, UK). Membrane was blocked for one hour using blocking solution containing Tris-buffered saline, 5\% milk and 1\% Tween. Primary antibody (clone H300, rabbit polyclonal TNC antibody; Santa-Cruz Biotechnology, Santa Cruz, CA, USA) was added and incubated for overnight at $4^{\circ} \mathrm{C}$. After subsequent washes, secondary antibody(donkey anti-rabbit horseradish peroxidase-linked IgG; Amersham Biosciences) was added and incubated for 1 hour. An ECL kit was used for detection of protein according to manufacturer's instructions and visualised using X-ray film (Xerox, USA) with a variety of exposure times.

\subsection{Analysis of Tumour Cell Invasion}

The cell culture inserts (FluoroBlok $8 \mu \mathrm{m}$ pore) were coated with $200 \mu \mathrm{l}$ of Matrigel (1:100 dilutions in a cold Opti-MEM) and incubated at $37{ }^{\circ} \mathrm{C} / 5 \% \mathrm{CO}_{2}$ for 2 hrs. Post-incubation, excess Matrigel was removed. Cells were stained with lipophilic fluorescent tracer dye 1,1'didodecyl-3,3,3',3'-tetramethylindocarbocyanine perchlorate (DiIC12(3)) in complete DMEM at a concentration of 10 $\mu \mathrm{g} / \mathrm{ml}$ and incubated for 1 hour at $37{ }^{\circ} \mathrm{C} / 5 \% \mathrm{CO}_{2}$. Cells were washed 3 times in DPBS and incubated for 1 hour in complete DMEM. Cells were then harvested and counted. An aliquot of fluorescently-stained cells were seeded into the insert in $200 \mu \mathrm{l}$ complete DMEM. $700 \mu \mathrm{l}$ of complete DMEM was then placed into each insert. Cells were incubated for $4 \mathrm{hr}$ at $37{ }^{\circ} \mathrm{C} / 5 \% \mathrm{CO}_{2}$ and the media in the insert was replaced with $200 \mu \mathrm{l}$ Opti-MEM [14]. Cells were cultured for $48 \mathrm{hr}$ at $37^{\circ} \mathrm{C} / 5 \% \mathrm{CO}_{2}$ in a FLUOstar OPTIMA plate reader (BMG Labtech, UK) with measurements taken every 2 hrs.

\subsection{Analysis of Tumour Cell Proliferation}

MDA-MB-231 cells transfected with siRNA targeting total TNC and TNC specific isoforms were cytospun at a cell density of $2 \times 10^{4}$ cells. The cells were fixed with $10 \%$ formalin for 10 mins. Immunocytochemistry was carried out using an antibody against Phospho-Histone H3 (pHH3) as an immunomarker specific for cells undergoing mitosis [19]. The cells were transfected with siRNAs and incubated for $24 \mathrm{hrs}$ in transfection media. Cells were then incubated with fresh complete media for 48 hrs. The expression of pHH-3 was analysed by immunocytochemistry using the Novolink polymer detection system and antipHH-3 antibody at a dilution of 1:1000. The analysis of pHH-3 staining was performed using the ImmunoRatio image analysis application, which calculates the percentage of positive staining [20].

\subsection{Statistical Analysis}

Statistical analysis was performed using GraphPad Prism 5 for Windows (GraphPad Software, Inc., USA). All assays used to determine the effects of siRNA employed a Two-Way Analysis of Variance (Two-way ANOVA) and Post-hoc Tukey's test in order to test the significant variance between cells transfected with TNC siRNAs compared to cells transfected to scrambled siRNA. All tests were two-sided and $\mathrm{P}<0.05$ was considered significant.

Table 3. Relative expression of total TNC and TNC isoforms in breast cancer cell lines

\begin{tabular}{|c|c|c|c|c|c|c|c|c|c|c|c|}
\hline \multirow{2}{*}{ Cell Line } & \multicolumn{6}{|c|}{ Number of TNC transcripts $\left(\times 10^{3}\right)$} & \multicolumn{5}{|c|}{ Percentage (\%) of total TNC transcripts } \\
\hline & Total TNC & TNC-S & TNC-AD1 & TNC-AD2 & TNC-9/16 & TNC-14/16 & TNC-S & TNC-AD1 & TNC-AD2 & TNC-9/16 & TNC-14/16 \\
\hline MCF-7 & 0 & 0 & 0 & 0 & 0 & 0 & 0 & 0 & 0 & 0 & 0 \\
\hline ZR-75-1 & 0 & 0 & 0 & 0 & 0 & 0 & 0 & 0 & 0 & 0 & 0 \\
\hline MDA-MB-231 & 5614.89 & 1714.16 & 425.26 & 245.41 & 193.10 & 1233.31 & 32.84 & 7.99 & 4.04 & 3.68 & 21.42 \\
\hline MDA-MB-436 & 2912.51 & 244.15 & 726.29 & 34.95 & 27.50 & 815.55 & 9.82 & 25.35 & 1.09 & 1.10 & 34.65 \\
\hline MDA-MB-468 & 1654.45 & 1938.75 & 72.57 & 6.02 & 95.16 & 281.05 & 118.97 & 4.79 & 0.36 & 5.58 & 18.36 \\
\hline HBL-100 & 14021.94 & 1481.73 & 2070.32 & 38.70 & 79.88 & 397.54 & 10.57 & 14.75 & 0.28 & 0.57 & 2.83 \\
\hline
\end{tabular}

\section{Results}

\subsection{Expression of Total TNC and TNC Isoforms in TNC Positive Cell Lines}

The native expression of TNC was first investigated in breast cancer cell lines. Expression of total TNC and TNC isoforms varied between cell lines which is explained in (Table 4). ER positive cells (MCF-7, ZR-75-1) did not express TNC (Table 3). MDA-MB-231 and HBL-100 showed the highest level of total TNC, whereas MDA- 
MB-468 and MDA-MB-436 expressed lower levels of total TNC. TNC-14/16 and TNC-AD1 were expressed in all total TNC positive cell lines, with MDA-MB-231 expressing the highest levels of TNC-14/16 $(>1,200,000$ molecules per $1 \mu \mathrm{g}$ RNA), and HBL-100 cells expressed the highest level of TNC-AD1 (>700,000 molecules per 1 $\mu \mathrm{g}$ RNA). MDA-MB-436 cells showed the highest percentages of high molecular weight TNC (AD1 and 14/16) (>21\% total TNC) (Table 3).

\subsection{The Effect of Knockdown of TNC mRNA Expression by siRNA}

RT-qPCR analysis showed that the siRNAs tested were shown to significantly reduce TNC mRNA expression (Figure 1A). SiRNAs targeting TNC at invariant exon 24 gave the most statistically significant knockdown of total
TNC ( $\leq \leq 0.001$ ) (Figure 1A). Highest knockdown of high MW TNC isoforms was achieved using siRNAs targeting exon 14 ( $p \leq 0.01$ and $p \leq 0.001$ ) (Figure $1 \mathrm{~A}$ ). Specifically, TNC-AD1 was down-regulated significantly by siRNA targeting exons 14-AD1 ( $\mathrm{p} \leq 0.001)$ (Figure 1A). Western blot analysis of cell lysates and conditioned media (Figure 1B), collected from cells transfected with siRNAs targeting total TNC and TNC high MW isoforms confirmed TNC down-regulation at the protein level. There was a specific reduction of the high MW TNC level caused by targeting exon 14 with no effect on truncated TNC levels. There was no observable change in TNC protein levels in cells transfected with siRNAs against exons 14-AD1, as might be expected as TNC-Long (TNC$\mathrm{L})$ and TNC-Short (TNC-S) are the predominant isoforms detected (Figure 1B).

\section{(A)}

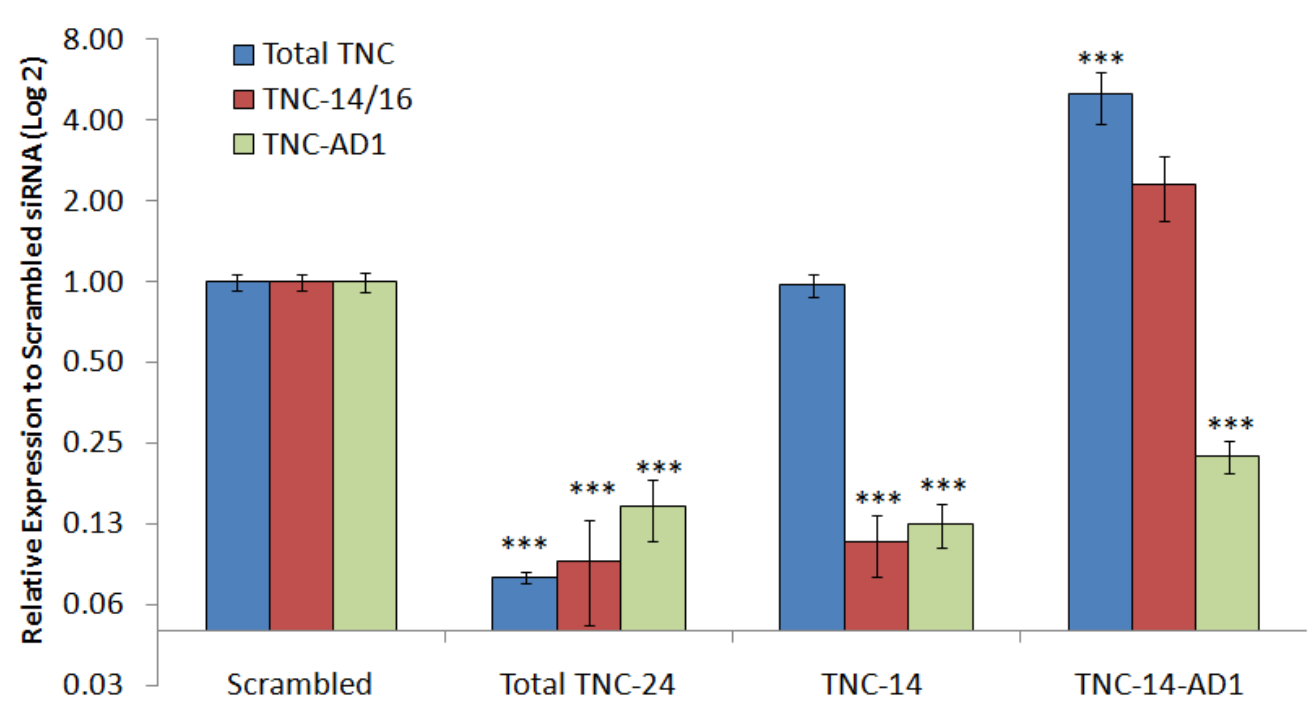

siRNA Treatment

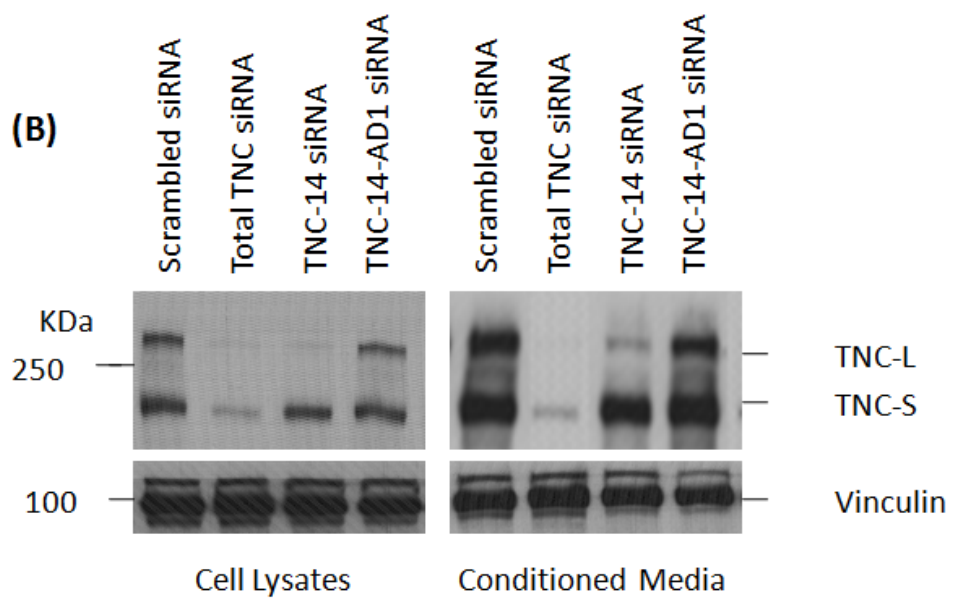

Figure 1. Efficiency of TNC knockdown by siRNA at mRNA and protein levels. (A) Real time PCR analysis shows the relative expression of total TNC and specific isoforms in transfected MDA-MB-231 cells with total TNC and high MW TNC siRNA compared to scrambled siRNA. The significant difference between TNC expression and scrambled siRNA are indicated by asterisk $(* * *=\leq 0.001)$. (B) Western blot analysis of the transfected MDA-MB-231 breast cancer cell lines in both cell lysate, and conditioned media. siRNAs targeting different exons are represented in cell lysates and conditioned media. Upper panel represents predominant TNC isoforms (TNC-L and TNC-S). Lower panel represents loading control (Vinculin) 


\subsection{Effects of TNC Knockdown on Breast Cancer Cell Invasion and Proliferation}

The MDA-MB-231 cells were analysed for cell invasion and proliferation profiles for 48 hours, which was preceded by siRNA transfection for 24 hours. The maximum reduction in the invasion and proliferation was observed in cells transfected with Total TNC. Two Dimension (2D) cell invasion assay showed statistically significant decrease in invasion compared to untransfected cells and cells transfected with a scrambled siRNA (Total TNC siRNA $p \leq 0.001$, TNC-14 siRNA $p \leq 0.001$ ) (Figure $2 \mathrm{~A} \& \mathrm{~B})$.

pHH-3 immunostaining of cells transfected with TNC siRNAs also showed a significant decrease in $\mathrm{pHH}-3$ expression compared to cells transfected with scrambled siRNA (total TNC siRNA $\mathrm{p} \leq 0.001$, TNC-14-AD siRNA $\mathrm{p} \leq 0.05)$ (Figure $2 \mathrm{C} \& \mathrm{D}$ ).
(A)

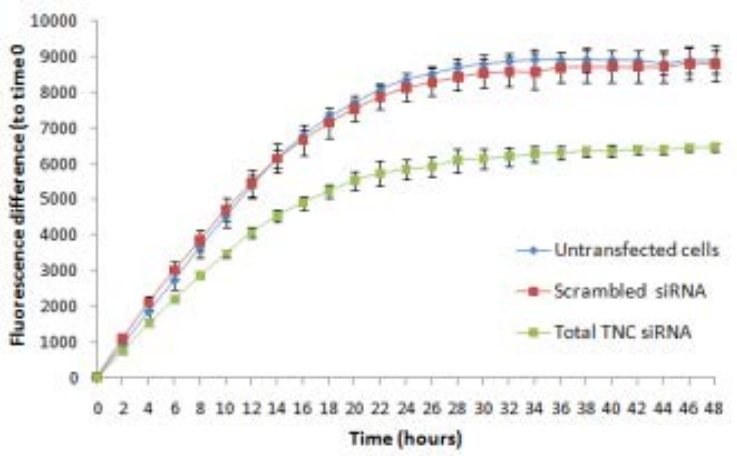

(C)

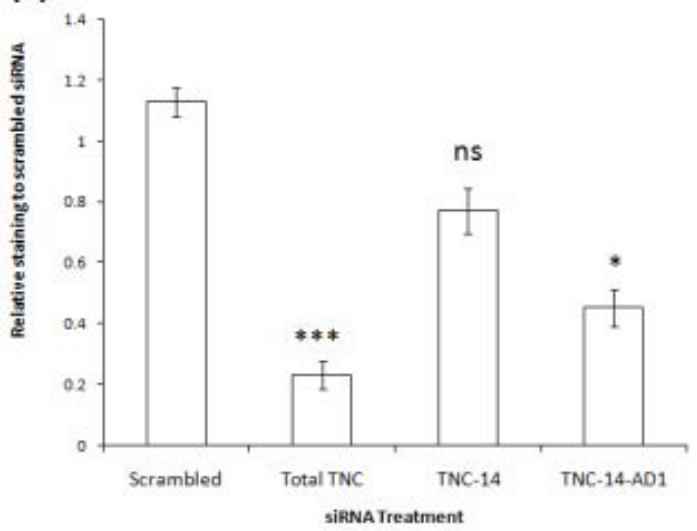

(B)

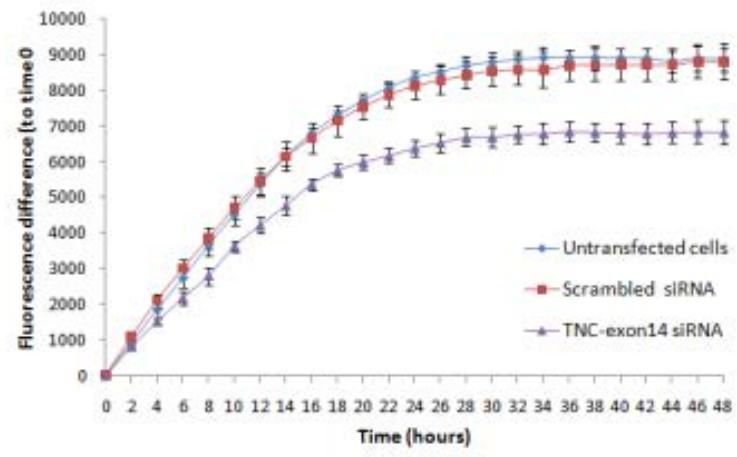

(D)
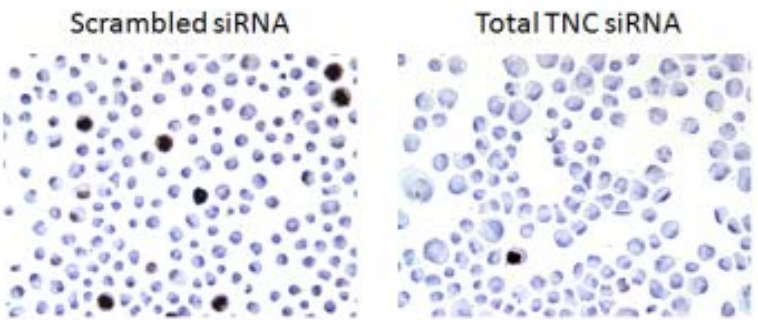

TNC-14 SIRNA

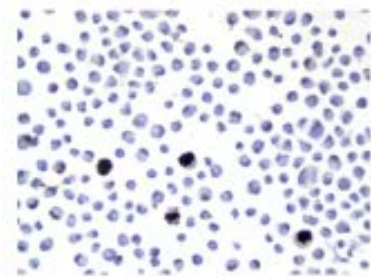

TNC-AD1 SIRNA

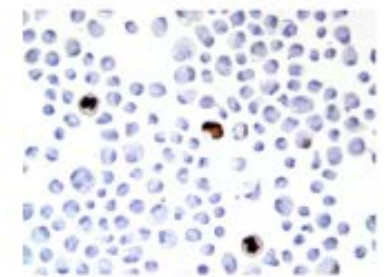

Figure 2. Effects of TNC knockdown on breast cancer cell invasion and proliferation. (A) 2D invasion assay for MDA-MB-231 cell line transfected with specific isoforms siRNA compared to untreated cells and cells treated with scrambled siRNA, using 5\% FCS DMEM as the chemotactic source. Fluorescence values were measured every 2 hours for 48 hours. Graphs represent the difference in fluorescence from time 0 . Error bars represents standard error of the mean (6 replicates). (C) Analysis of pHH-3 staining on MDA-MB-231 transfected with total TNC and specific isoforms siRNAs. The graph was generated based on the ImmunoRatio image analysis application, which calculate the percentage of pHH-3 positive staining within five fields. This experiment was performed in triplicate, and the graph shows the mean of five fields (approximately 200 cells per field). (D) Immunocytochemistry staining of pHH-3 expression. MDA-MB-231 cells were transfected in triplicate with total TNC and specific isoforms siRNAs (Magnification is x40). All statistical tests were Two-way ANOVA and Posthoc Tukey test between siRNA treatments and time in cells transfected with siRNAs compared to the cells transfected with scrambled siRNA

\section{Discussion}

Our study had shown that TNC plays a pivotal role in breast cancer pathogenesis. The TNC knockdown in breast cancer cell lines revealed significant decrease in proliferation and invasion which is consistent with previous studies done by our group. Previous studies in our group have shown differences in the pattern of TNC isoform expression between benign, pre-invasive and invasive lesions using RT-qPCR and Southern blotting. Furthermore, all types of lesions were shown to express truncated tenascin (tTN), and TNC isoforms containing exon 16 (TNC16) and exon 14/16 (TNC14/16) [12]. In this study, siRNAs targeting different TNC exons were transfected into the highly invasive MDA-MB-231 cells and the results obtained showed a significant down regulation of TNC expression at both the mRNA and protein levels, as demonstrated by RT-qPCR and Western blotting, respectively. Previous studies by our group have demonstrated that high MW isoforms of TNC facilitate tumour invasion and proliferation in breast cancer $([12,13,14])$, with an increase in invasion seen in MDAMB-231, MCF-7, T-47D and GI-101 cell lines overexpressing TNC 9/16 and TNC 9/14/16 [13]. In this 
regard, MDA-MB-231 cell lines were selected and transfected with TNC siRNAs in order to assess the effects of TNC knockdown on cell invasion and proliferation. The results obtained in this study correlate with previous findings by confirming that TNC plays an important role in the pathogenesis of breast cancer by the significant reduction in tumour invasion and proliferation when TNC expression is down-regulated. TNC-16 and TNC 14/16 have been associated with tumour cell malignancy, but TNC 14/16 was specific for invasion [12], as well as promotion of tumour cell proliferation and invasion in a MMP independent and dependent mechanism [13]. This study showed that knocking down expression of isoforms containing exon 14 decreases cancer cell invasion and proliferation, which substantiates previous studies by suggesting that high MW isoforms are strongly associated with invasion and proliferation. The importance of the full length TNC isoform in the progression of breast cancer has been previously shown to be at invading sites of intra-ductal cancers and in the stroma of invasive ductal cancers [21]. Moreover, additional exons (exon 15 in domain C) have been reported to be associated with vessels of anaplastic glioma and considered as a marker of vascular proliferation [22]. Despite all this, the role of TNC containing AD1 repeats in breast cancer progression is not clear. TNC containing novel $\mathrm{AD} 1$ and $\mathrm{AD} 2$ repeats were first identified in chicken embryo and later identified in normal and malignant human oral mucosa $([23,24])$. The AD1 repeat was also identified in fibrosarcoma cell line, malignant melanoma and ductal breast carcinoma [25]. Extensive RT-qPCR analysis of breast tissue from high grade and hormone sensitive breast cancer in young women has shown novel TNC isoforms containing AD1 repeats. Furthermore, 2D invasion assays have shown that AD1 containing isoforms influence cell invasion in proportion to the TNC14/16 isoform [14]. The results obtained here correlate with previous studies in that siRNAs targeting total TNC, TNC-14 and TNC-14-AD1 resulted in a significant decrease in cancer cell proliferation and invasion.

\section{Conclusion}

Targeted silencing of TNC expression using siRNA technology leads to an efficient reduction of TNC expression with significant effects on invasion and proliferation in breast cancer cell lines endogenously expressing TNC. This study showed that high MW TNC isoforms may play an important role in breast cancer progression and warrants further investigation to elucidate the pathways on which they act.

\section{Acknowledgment}

I would like to thank Professor Jacqui Shaw, Dr Howard Pringle and Dr David Guttery (University of Leicester, UK) for advice and generous support.

\section{Abbreviations}

Tenascin-C (TNC), Extracellular Matrix (ECM), Molecular Weight (MW), Small Interfering RNA (siRNA), Phispho-Histone H3 (pHH3), Matrix metalloproteinases
(MMPs), Reverse Transcription (RT),Polymerase Chain Reaction (PCR), Additional Domain 1 (AD1), Oestrogen receptor (ER), TNC-Long (TNC-L), TNC-Short (TNC-S), Two Dimension (2D).

\section{References}

[1] ZiOi, Tao, Shi Aimin, Lu Cuntao, Song Tao, Zhao Jing. 2014 "Breast Cancer: Epidemiology and Etiology", Cell biochemistry and biophysics.

[2] Orend G, Saupe F, Schwenzer A, Midwood K. "The extracellular matrix and cancer: regulation of tumor cell biology by tenascin-C". iConcept Press 2014. p. 1-139.

[3] Pickup MW, Mouw JK, Weaver VM. The extracellular matrix modulates the hallmarks of cancer. EMBO Rep 2014; 15(12):1243-53; PMID:25381661

[4] Jones, P.L. 2001, "Extracellular matrix and tenascin-C in pathogenesis of breast cancer", Lancet, vol. 357, no. 9273, pp. 1992-1994.

[5] Ioachim E, Charchanti A, Briasoulis E, Karavasilis V, Tsanou H, Arvanitis DL, Agnantis NJ, Pavlidis N: 2002," Immunohistochemical expression of extracellular matrix components tenascin, fibronectin, collagen type IV and laminin in breast cancer: their prognostic value and role in tumour invasion and progression", Eur J Cancer, vol. 38, pp. 2362-2370.

[6] Midwood, K.S. \& Orend, G. 2009, "The role of tenascin-C in tissue injury and tumorigenesis.", Journal of cell communication and signaling, vol. 3, no. 3-4, pp. 287-310.

[7] Joester, A. \& Faissner, A. 2001, "The structure and function of tenascins in the nervous system", Matrix Biology, vol. 20, no. 1, pp. 13-22.

[8] Orend, G. 2005, "Potential oncogenic action of tenascin-C in tumorigenesis", International Journal of Biochemistry \& Cell Biology, vol. 37, no. 5, pp. 1066-1083.

[9] Jahkola, T., Toivonen, T., Nordling, S., von Smitten, K. \& Virtanen, I. 1998a, "Expression of tenascin-C in intraductal carcinoma of human breast: Relationship to invasion", European journal of cancer, vol. 34, no. 11, pp. 1687-1692.

[10] Ishihara, A., Yoshida, T., Tamaki, H. \& Sakakura, T. 1995, "Tenascin expression in cancer cells and stroma of human breast cancer and its prognostic significance", Clinical Cancer Research, vol. 1, no. 9, pp. 1035-1041.

[11] Chiquet-Ehrismann, R. \& Chiquet, M. 2003, "Tenascins: regulation and putative functions during pathological stress", Journal of Pathology, vol. 200, no. 4, pp. 488-499.

[12] Adams, M., Jones, J.L., Walker, R.A., Pringle, J.H. \& Bell, S.C. 2002, "Changes in tenascin-C isoform expression in invasive and preinvasive breast disease", Cancer research, vol. 62, no. 11, pp. 3289.

[13] Hancox, R.A., Allen, M.D., Holliday, D.L., Edwards, D.R., Pennington, C.J., Guttery, D.S., Shaw, J.A., Walker, R.A., Pringle, J.H. \& Jones, J.L. 2009, "Tumour-associated tenascin-C isoforms promote breast cancer cell invasion and growth by matrix metalloproteinase-dependent and independent mechanisms", Breast Cancer Research, vol. 11, no. 2.

[14] Guttery, D.S., Hancox, R.A., Mulligan, K.T., Hughes, S., Lambe, S.M., Pringle, J.H., Walker, R.A., Jones, J.L. \& Shaw, J.A. 2010a, "Association of invasion-promoting tenascin-C additional domains with breast cancers in young women", Breast Cancer Research, vol. 12, no. 4, pp. R57.

[15] Tavazoie, S.F., Alarcon, C., Oskarsson, T., Padua, D., Wang, Q., Bos, P.D., Gerald, W.L. \& Massague, J. 2008, "Endogenous human microRNAs that suppress breast cancer metastasis", Nature, vol. 451, no. 7175, pp. 147-U3.

[16] Zukiel, R., Nowak, S., Wyszko, E., Rolle, K., Gawronska, I., Barciszewska, M.Z. \& Barciszewski, J. 2006, "Suppression of human brain tumor with interference RNA specific for tenascin-C", Cancer Biology \& Therapy, vol. 5, no. 8, pp. 1002-1007.

[17] Hirata, E., Arakawa, Y., Shirahata, M., Yamaguchi, M., Kishi, Y., Okada, T., Takahashi, J.A., Matsuda, M. \& Hashimoto, N. 2009, "Endogenous tenascin-C enhances glioblastoma invasion with reactive change of surrounding brain tissue", Cancer Science, vol. 100, no. 8, pp. 1451-1459.

[18] Fukunaga-Kalabis, M., Martinez, G., Nguyen, T.K., Kim, D., Santiago-Walker, A., Roesch, A. \& Herlyn, M. 2010, "Tenascin-C 
promotes melanoma progression by maintaining the ABCB5positive side population", Oncogene, vol. 29, no. 46, pp. 61156124.

[19] Veras, E., Malpica, A., Deavers, M.T. \& Silva, E.G. 2009, "Mitosis-specific Marker Phospho-histone H3 in the Assessment of Mitotic Index in Uterine Smooth Muscle Tumors: A Pilot Study", International Journal of Gynecological Pathology, vol. 28, no. 4, pp. 316-321.

[20] Tuominen, V.J., Ruotoistenmaki, S., Viitanen, A., Jumppanen, M. \& Isola, J. 2010, "ImmunoRatio: a publicly available web application for quantitative image analysis of estrogen receptor (ER), progesterone receptor (PR), and Ki-67", Breast Cancer Research, vol. 12, no. 4, pp. R56.

[21] Tsunoda, T., Inada, H., Kalembeyi, I., Imanaka-Yoshida, K., Sakakibara, M., Okada, R., Katsuta, K., Sakakura, T., Majima, Y. \& Yoshida, T. 2003, "Involvement of large tenascin-C splice variants in breast cancer progression", American Journal of Pathology, vol. 162, no. 6, pp. 1857-1867.
[22] Viale, G.L., Castellani, P., Dorcaratto, A., Pau, A., Sehrbundt, E., Siri, A., Biro, A. \& Zardi, L. 2002, "Occurrence of a glioblastomaassociated tenascin-C isoform in cerebral cavernomas and neighboring vessels", Neurosurgery, vol. 50, no. 4, pp. 838-842.

[23] Sriramarao, P. \& Bourdon, M.A. 1993a, "A Novel Tenascin TypeIii Repeat is Part of a Complex of Tenascin Messenger-Rna Alternative Splices", Nucleic acids research, vol. 21, no. 1, pp. 163-168.

[24] Mighell, A.J., Thompson, J., Hume, W.J., Markham, A.F. \& Robinson, P.A. 1997, "Human tenascin-C: Identification of a novel type III repeat in oral cancer and of novel splice variants in normal, malignant and reactive oral mucosae", International Journal of Cancer, vol. 72, no. 2, pp. 236-240.

[25] Derr, L.B., Chiquet-Ehrismann, R., GandourEdwards, R., Spence, J. \& Tucker, R.P. 1997, "The expression of tenascin-C with the $\mathrm{AD} 1$ variable repeat in embryonic tissues, cell lines and tumors in various vertebrate species", Differentiation, vol. 62, no. 2, pp. 7182. 